\title{
Efficacy of Yan-Shen capsule on advanced chronic kidney disease (CKD) patients: An open, randomized, prospective, comparative study
}

\author{
Fa-Huan Yuan*", Wei-Ping Hou\#, Li-Xia Guang, Xiang Du and Rui Zhang \\ Department of Nephrology, Xinqiao Hospital, Chongqing 400037, P.R. China.
}

Accepted 19 October, 2012

\begin{abstract}
This study aimed to investigate the efficacy of Yan-Shen capsule, a traditional Chinese medicine compound, on patients with advanced chronic kidney disease (CKD). A randomized prospective clinical trial was conducted to investigate the efficacy of Yan-Shen capsule on hospital patients with advanced CKD (stages III to IV). Each group included 82 patients (males and females), and all patients were followed up every month for three years. From the Yan-Shen capsule group, 13 patients (16\%) reached the endpoints of the study, $64(78 \%)$ continued at stage IV or below, and 5 were not followed up. From the Niao-Du-Qing granules group, 18 patients $(22 \%)$ reached the endpoints, $60(73 \%)$ continued at stage IV or below, and 4 were not followed up. From the placebo group, 46 patients $(56 \%)$ reached the endpoints, $30(37 \%)$ continued at stage IV, and 6 were not followed up. As for the patients who reached the endpoints, a significant difference existed between the Yan-Shen capsule group and the placebo group $(P<0.05)$, but no significant difference was observed between the Yan-Shen capsule group and the Niao-Du-Qing granules group $(P>0.05)$. Annual serum creatinine $(\mathrm{SCr})$ increase, glomerular filtration rate (GFR) decrease, and erythropoietin requirement of the Yan-Shen capsule group were much lower than those of the placebo or Niao-Du-Qing granules group. Both Yan-Shen capsule and Niao-Du-Qing granules had significant protective effects on residual renal function in advanced CKD and were able to delay the progression to dialysis without obvious side effects. Yan-Shen capsule may be superior to Niao-Du-Qing granules in terms of protecting residual renal function and modifying anaemia.
\end{abstract}

Key words: Chronic kidney disease (CKD), residual renal function, therapy, traditional Chinese medicine.

\section{INTRODUCTION}

Chronic kidney disease (CKD) incidence has risen to as high as 10\% (Thorp et al., 2006; Zhang et al., 2006). Many CKD patients will progress to end-stage renal disease (ESRD) and require dialysis or kidney transplanttation. However, dialysis and kidney transplantation have disadvantages such as high price, inconvenience, and side effects. Other issues affect the treatment of kidney

*Corresponding author. Email: fahuanyuan@126.com. Tel: 86 13708350570. Fax: 86-02368774321.

\# These authors contributed equally to the work. disease, such as the shortage of donor kidneys for transplantation, chronic rejection and side effects of antirejection drugs, and a higher risk of complications for patients who are undergoing peritoneal dialysis or haemodialysis for a prolonged time. In addition, the mortality rate of chronic haemodialysis patients remains high despite significant improvements in dialysis technology (Hamlett and Haragsim, 2007).

Nephrologists seek to determine how CKD progression towards ESRD can be delayed and to elevate the quality of life of CKD patients. Recently, some studies indicated that many natural plant extracts had anti-inflammatory and anti-fibrosis effects in some chronic diseases (Sima et al., 2012; Jinous and Fereshteh, 2012; Muhammad et 
al., 2012) and our previous experiments proved that YanShen capsules could inhibit fibrosis of the remnant kidney of five out of six nephrectomized rats and retard the progression of chronic renal failure (CRF) without obvious side effects (Yuan et al., 1998, 2004).

Yan-Shen capsules, which contain up to 10 kinds of Chinese herbs such as Astragali radix, ligustrazine, rhubarb, Cordyceps sinensis, and ginseng, could delay the worsening of kidney functions through multiple protective mechanisms. These capsules, which have been used as self-made traditional Chinese medicine compounds in treating CKD patients in our hospital for 15 years, could significantly improve clinical symptoms. The Chinese patent for Yan-Shen capsules has already been obtained (patent no. ZL2003101109342). To further investigate the effects of Yan-Shen capsules and compare them with those of Niao-Du-Qing granules, an open, randomized, prospective clinical trial which involved advanced CKD (stage III to IV) patients was conducted.

\section{MATERIALS AND METHODS}

\section{Inclusion criteria}

Based on the diagnosis criteria of the K/DOQI clinical practice guidelines for chronic kidney disease (National Kidney Foundation, 2002), we screened 982 advanced CKD patients (males and females, stage III to IV) who did not need corticosteroid or angiotensin-converting-enzyme inhibitor (ACEI) treatments and who were admitted to our hospital from January 2004 to 31 December, 2005. The main causes of CKD include chronic glomerulonephritis, lupus nephritis, and autosomal dominant polycystic kidney disease. The study was conducted at the Renal Division of Xinqiao Hospital, and the study protocol was approved by the Xinqiao Ethics Committee.

\section{Exclusion criteria}

Exclusion criteria include the following: renal tubulointerstitial, blood vessel, and infection diseases, renal tumour, obstructive and diabetic nephropathy, hypertension-induced kidney disease, generous proteinuria (> $3.5 \mathrm{~g} /$ day), heart, liver, or lung dysfunction, recent fever or infection, tumour, hyperthyreosis, hypothyroidism, hyperkalemia, severe oedema, uncontrolled hypertension, and haemoglobin $(\mathrm{Hb})<80 \mathrm{~g} / \mathrm{L} ;<18$ or $>70$ years old; not suited for conservative treatment and follow-up.

\section{Endpoints of observation}

\section{Primary endpoint}

The patients progressed to ESRD (that is, with serum creatinine $(\mathrm{SCr})>707 \mu \mathrm{mol} / \mathrm{L}(8 \mathrm{mg} / \mathrm{dl})$ or glomerular filtration rate $(\mathrm{GFR})<$ $10 \mathrm{ml} / \mathrm{min} / 1.73 \mathrm{~m}^{2}$

\section{Secondary endpoints}

The patients contracted myocardial infarction, stroke, generous gastrointestinal haemorrhage, severe infection, trauma, tumour, severe hyperpotassemia or acidosis, or uncontrolled hypertension, thereby requiring the use of $\mathrm{ACEI}$ and/or angiotensin II receptor blocker (ARB).

\section{Grouping}

Based on the inclusion and exclusion criteria, 246 patients (males and females) were selected and enrolled into the study. These patients were randomly divided into three groups by using block randomization, with a block size of six. The Yan-Shen capsules group (group $\mathrm{I}, \mathrm{n}=82$ ) patients orally took Yan-Shen capsules (powdered extracts, $0.45 \mathrm{~g} /$ capsule, 5 capsules/time, 3 times/day). The Niao-Du-Qing granules group (group II, $\mathrm{n}=82$ ) patients orally took the traditional Chinese medicine compound Niao-Du-Qing granules produced by Guangzhou Kangchen Medicine (national medicine permission number: Z10970122), which could facilitate the excretion of urotoxins and improve energy metabolism (granules, $5 \mathrm{~g} /$ pocket, 1 pocket/time, 3 times/day). The negative control group was the placebo group (group III, $\mathrm{n}=82$ ). The patients from each group were given antihypertensive agents (except ACEI and ARB), sodium bicarbonate, and $1 \alpha$ hydroxyl vitamin D3 according to the patient's condition. All patients ate a low-protein diet $(0.8 \mathrm{~g} / \mathrm{d} / \mathrm{kg})$ without keto acids or steroids. The patients who had lower $\mathrm{Hb}(<90 \mathrm{~g} / \mathrm{L})$ were given erythropoietin $(100 \mathrm{U} / \mathrm{kg}$, subcutaneous injections, 1 to 2 times/week) and intravenous ferralium (dosage based on the assay results of iron metabolism) to maintain $\mathrm{Hb}$ within the 90 to $120 \mathrm{~g} / \mathrm{L}$ range. All patients were discharged from the hospital after two to three weeks and were then followed up through monthly outpatient visits for three years. The observations ended on 31 December, 2008.

\section{Observations}

The patients' mental status, appetite, urine volume, stool, and blood pressure were observed and recorded by physicians every month. $\mathrm{SCr}$ (enzyme method), blood urea nitrogen (BUN, enzyme method), serum potassium $\left(\mathrm{K}^{+}\right)$, bicarbonate $\left(\mathrm{HCO}^{3-}\right)$, and liver function, including plasma albumin, pre-albumin, alanine transaminase, glutamic-oxalacetic transaminase, globulin, total bilirubin, direct bilirubin, and indirect bilirubin, were measured by using an automatic biochemistry analyzer. $\mathrm{Hb}$ was measured by using an automatic blood cell analyzer. The assays were carried out in the central clinical laboratory of our hospital. GFR was calculated based on the modification of diet in renal disease (MDRD) formula (Levey et al., 2007): GFR $\left(\mathrm{ml} / \mathrm{min} / 1.73 \mathrm{~m}^{2}\right)=30849 \times(\mathrm{SCr} \mu \mathrm{mol} / \mathrm{L})-1.154$ $\times$ age -0.203 (if female $\times 0.742$ ). Some patients with a short history of the disease and normal-size kidneys received renal biopsy. The ratios of sclerotic glomeruli and damaged renal interstitium were calculated (Yuan et al., 2004).

\section{Statistical analysis}

Continuous variables were presented as mean plus standard deviation (mean $\pm S$ ) and compared by using unpaired two-way analysis of variance (ANOVA). Discrete variables were compared by using chi-square test. Statistical analysis was performed by using the Statistics Package for Social Science 13.0 software for Windows. $P$ values of $<0.05$ were considered significant.

\section{RESULTS}

\section{Patient condition while enrolled}

The patient condition of the three groups was comparable 
with respect to age, gender, primary disease, blood pressure, urine volume, $\mathrm{Hb}$, renal function, and renal pathology (Table 1 to 4 ).

\section{Patients reaching endpoints}

Overall, throughout the three years of observation, 13 patients (16\%) in group I reached the endpoints (11 started dialysis, 2 used ACEI and ARB), 64 (78\%) remained at or below stage IV CKD, and $5(6 \%)$ were lost to follow-up. In group II, 18 patients $(22 \%)$ reached the end points (15 started dialysis, 3 patients used ACEI and ARB), $60(73 \%)$ remained at or below stage IV CKD, and $4(5 \%)$ were lost to follow-up. In group III, 46 patients (56\%) reached the endpoints (38 started dialysis, 8 patients used ACEI and ARB), 30 patients (37\%) remained at or below stage IV CKD, and $6(7 \%)$ were lost to follow-up. In group I, the patients who reached the endpoints were fewer than those in group II although the difference was not statistically significant $(P>0.05)$ and were much fewer than those of group III, with a statistically significant difference $(P<0.05)$ (Table 5$)$. The main reason why patients reached the endpoints was so they could begin dialysis; other minor reasons were the use of ACEI and ARB. No patients reached the endpoint as a result of myocardial infarction, heart failure, stroke, gastrointestinal haemorrhage, severe infection, trauma, tumour, or acidosis. No significant difference exists between the numbers of patients from each group who were not followed up $(P>0.05)$.

\section{General condition of patients during observation}

In the three groups, the patients who did not reach the endpoints had good general condition, including normal mental status and appetite, and no cardiovascular or cerebrovascular events or gastrointestinal haemorrhage. Nine patients in group I, 12 in group II, and 8 in group III had diarrhoea during the observation period, and no significant difference $(P>0.05)$ was observed among them. The patients in the three groups who did not reach the endpoints had no hyperkalemia, acidosis, or liver dysfunction. The Yan-Shen capsule group had significantly fewer patients who needed erythropoietin due to low $\mathrm{Hb}(<90 \mathrm{~g} / \mathrm{L})$ than the placebo group and the NiaoDu-Qing granules group $(P<0.05)$ (Table 6$)$. The patients in the three groups excluded other causes of anaemia, such as gastrointestinal diseases, bleeding, and tumour.

\section{Renal function and blood pressure variation}

In the three groups, $\mathrm{SCr}$ and BUN increased, and GFR decreased as time passed (Table 7 to 9). A smaller annual increase of SCr $(25.7 \mu \mathrm{mol} / \mathrm{L})$ occurred in group I compared with that in group II $(41.7 \mu \mathrm{mol} / \mathrm{L})$ and in group
III $(116.4 \mu \mathrm{mol} / \mathrm{L})$. At the end of the third year, the $\mathrm{SCr}$ of group I $(282 \pm 68.3 \mu \mathrm{mol} / \mathrm{L})$ was significantly lower than that of group II $(318 \pm 73.9 \mu \mathrm{mol} / \mathrm{L}, \mathrm{P}<0.05)$ and that of group III $(544 \pm 91.3 \mu \mathrm{mol} / \mathrm{L}, \mathrm{P}<0.01)$. The annual BUN increases of group I $(2.2 \mathrm{mmol} / \mathrm{L})$ and group II $(2.0 \mathrm{mmol} / \mathrm{L})$ were so close that no significant difference in BUN existed between group I $(19.2 \pm 9.3 \mathrm{mmol} / \mathrm{L})$ and group II $(18.3 \pm 9.9 \mathrm{mmol} / \mathrm{L}, \mathrm{p}>0.05)$ at the end of the third year. The annual BUN increase in group III $(4.7 \mathrm{mmol} / \mathrm{L})$ was significantly higher than those of groups I and II $(\mathrm{P}<0.05)$. The BUN of group III $(26.1 \pm 9.3 \mathrm{mmol} / \mathrm{L})$ at the end of the third year was much higher than that of groups I and II $(\mathrm{P}<$ 0.05). Group I had a smaller annual GFR decrease (3.4 $\left.\mathrm{ml} / \mathrm{min} / 1.73 \mathrm{~m}^{2}\right)$ than that of group II $\left(5.1 \mathrm{ml} / \mathrm{min} / 1.73 \mathrm{~m}^{2}\right)$ and group III $\left(7.2 \mathrm{ml} / \mathrm{min} / 1.73 \mathrm{~m}^{2}\right)$. The GFR of group I $\left(23.1 \pm 4.3 \mathrm{ml} / \mathrm{min} / 1.73 \mathrm{~m}^{2}\right)$ was much higher than that of group II $\left(20.1 \pm 4.9 \mathrm{ml} / \mathrm{min} / 1.73 \mathrm{~m}^{2}, \mathrm{P}<0.05\right)$ and group III $\left(14.4 \pm 3.7 \mathrm{ml} / \mathrm{min} / 1.73 \mathrm{~m}^{2}, \mathrm{P}<0.05\right)$ at the end of the third year. The ratio and mean blood pressure of the hypertension patients in three groups were similar $(P>0.05)$ (Table 10).

\section{DISCUSSION}

The issue of delaying CKD progression to ESRD is a medical and social problem that concerns both nephrologists and patients. Solving this problem has tremendous scientific significance and economic benefit. Evidencebased clinical studies proved that ACEI and ARB could delay CKD progression to ESRD through its lowering effect on systemic and intracapsular blood pressure and its possible anti-inflammation and anti-proliferation effects (Hou et al., 2006; Enyu, 2006; Philipneri et al., 2008). However, ACEI and ARB could not promote the excretion of urotoxins and improve uremic symptoms. Chinese traditional medicines such as rhubarb and its compound preparation could delay CKD progression (Zhu and Wang, 2005; Guo et al., 2001; Wu et al., 2004).

Niao-Du-Qing granules are an extensively used and effective traditional Chinese medicine compound which contains rhubarb and can promote the excretion of urotoxins, decrease $\mathrm{SCr}$ and $\mathrm{BUN}$, and significantly improve uremic symptoms. Therefore, in this open, randomized, prospective, comparative study, Niao-DuQing granules were selected as a positive control to investigate the effect of Yan-Shen capsule on advanced CKD patients. The placebo control was set in our study. Yan-Shen capsule is a Chinese medicine compound that contains up to 10 kinds of herbs such as $A$. radix (a principal element), ligustrazine, rhubarb, $C$. sinensis, and ginseng (a contrary adjuvant).

A. radix contains astragalosides and astragalus polysaccharides, and its various biological actions such as immunomodulating activity and antioxidant and antiinflammatory properties offer health benefits. It also protects the kidney against ischemia (Cai et al., 2001). Ligustrazine is a strong scavenger of oxygen-free 
Table 1. The basic clinical characteristics of the three groups' patients.

\begin{tabular}{lccccccccc}
\hline \multirow{2}{*}{ Group } & \multicolumn{3}{c}{ Age (years) } & \multicolumn{2}{c}{ Gender } & \multicolumn{3}{c}{ Primary disease } \\
\cline { 2 - 10 } & Min & Max & Mean \pm SD & Male & Female & CGN & LN & ADPKD & Others \\
\hline I $(\mathrm{n}=82)$ & 18 & 70 & $40.3 \pm 10.1$ & 46 & 36 & 59 & 11 & 6 & 6 \\
II $(\mathrm{n}=82)$ & 19 & 70 & $41.3 \pm 9.6^{*}$ & $43^{*}$ & $39^{*}$ & $61^{*}$ & $10^{*}$ & $5^{*}$ & $6^{*}$ \\
III $(\mathrm{n}=82)$ & 20 & 69 & $43.1 \pm 12.4^{\#}$ & $47^{\#}$ & $35^{\#}$ & $63^{\#}$ & $8^{\#}$ & 4 & $7^{\#}$ \\
\hline
\end{tabular}

${ }^{*} \mathrm{P}>0.05$ (group I versus group II or group I versus group III). Group I: Yan-Shen Capsules Group; group II: Niao-Du-Qing granules group; group III: placebo group. CGN: chronic glomerulonephritis. LN: lupus nephritis. ADPKD: autosomal dominant polycystic kidney disease.

Table 2. Blood pressure and urine volume of three group patients.

\begin{tabular}{lccccc}
\hline Group & $\begin{array}{c}\text { Patients with } \\
\text { normal BP }\end{array}$ & $\begin{array}{c}\text { Patients with } \\
\text { hypertension }\end{array}$ & \multicolumn{2}{c}{ BP of hypertension patients (mmHg) } & $\begin{array}{c}\text { Urine volume } \\
\text { (ml/24 h) }\end{array}$ \\
\hline I $(\mathrm{n}=82)$ & 34 & 48 & $164.5 \pm 28.2$ & $104.1 \pm 13.3$ & $1410 \pm 312$ \\
II $(\mathrm{n}=82)$ & $37^{*}$ & $45^{*}$ & $165.4 \pm 25.9^{*}$ & $103.9 \pm 15.7^{*}$ & $1399 \pm 283^{*}$ \\
$\mathrm{III}(\mathrm{n}=82)$ & $36^{\#}$ & $46^{\#}$ & $162.4 \pm 26.4^{\#}$ & $101.9 \pm 13.4^{\#}$ & $1466 \pm 304^{\#}$ \\
\hline
\end{tabular}

${ }^{*} \mathrm{P}>0.05$ (group I versus group II or group I versus group III). Group I: Yan-Shen capsules group; group II: Niao-Du-Qing granules group; group III: placebo group. BP: blood pressure. SBP: systolic blood pressure. DBP: diastolic blood pressure.

Table 3. Renal function and $\mathrm{Hb}$ of three group patients.

\begin{tabular}{|c|c|c|c|c|}
\hline Group & $\mathrm{SCr}(\mu \mathrm{mol} / \mathrm{L})$ & BUN(mmol/L) & GFR $\left(\mathrm{ml} / \mathrm{min} / 1.73 \mathrm{~m}^{2}\right)$ & $\mathrm{Hb}(\mathrm{g} / \mathrm{L})$ \\
\hline I $(\mathrm{n}=82)$ & $205.6 \pm 42.1$ & $12.5 \pm 4.3$ & $33.4 \pm 4.2$ & $97.5 \pm 12.4$ \\
\hline$I I(n=82)$ & $193.2 \pm 43.5^{\star}$ & $12.2 \pm 4.4^{*}$ & $35.5 \pm 4.9^{*}$ & $99.3 \pm 13.1^{*}$ \\
\hline III $(\mathrm{n}=82)$ & $197.8 \pm 45.4^{\#}$ & $11.9 \pm 3.9^{\#}$ & $36.3 \pm 5.2^{\#}$ & $101.2 \pm 14.1^{\#}$ \\
\hline
\end{tabular}

*,\# $\mathrm{P}>0.05$ (group I versus group II or group I versus group III). Group I: Yan-Shen capsules group; group II: Niao-Du-Qing granules group; group III: placebo group.

Table 4. Renal pathology and injury degree of three group patients.

\begin{tabular}{ccccccccc}
\hline \multirow{2}{*}{ Group } & \multirow{2}{*}{$\mathbf{n}$} & \multicolumn{4}{c}{ Renal pathology } & \multicolumn{2}{c}{ Degree of injury (\%) } \\
\cline { 3 - 8 } & & IgAN & MePG & LN & Others & Ratio of sclerotic glomeruli & Damaged renal interstitium \\
\hline I & 64 & 22 & 23 & 8 & 11 & $65.3 \pm 8.7$ & $69.4 \pm 7.6$ \\
II & $61^{*}$ & $20^{*}$ & $23^{*}$ & $9^{*}$ & $9^{*}$ & $59.7 \pm 7.9^{*}$ & $61.3 \pm 7.8^{*}$ \\
III & $59^{\#}$ & $19^{\#}$ & $20^{\#}$ & $10^{\#}$ & $10^{\#}$ & $62.4 \pm 6.4^{\#}$ & $63.6 \pm 7.1^{\#}$ \\
\hline
\end{tabular}

\footnotetext{
${ }^{*} \mathrm{P}>0.05$ (group I versus group II, or group I versus group III). Group I: Yan-Shen capsules group; group II: Niao-Du-Qing granules group; group III: placebo group. IgAN: IgA glomerulonephritis. MePG: non-IgA mesangial proliferative glomerulonephritis. LN: lupus nephritis.
}

radicals and can reduce peroxide production. Rhubarb can increase toxin excretion from the gastrointestinal tract, improve metabolism, and suppress cell proliferation and extracellular matrix synthesis (Zheng et al., 2008). In addition, some studies show that $C$. sinensis and ginseng can help to alleviate proteinuria and hematuria, reduce immunocomplex in circulation, and alleviate glomerular injury mediated by immunity (Chen et al., 2009). The abovementioned characteristics of the compound herbs in the Yan-Shen capsule may be responsible for its protective effect on kidney function.

To reduce disturbance as much as possible, patients with organ damage in addition to that of the kidney or have generous proteinuria (which indicates that most of them need steroids) were excluded from the study. Table 1 to 4 show that no significant differences in age, gender, primary disease, blood pressure, urine volume, $\mathrm{Hb}$, renal pathology, and function existed in the three groups' patients upon enrolment, which proves that the general condition, clinical manifestation, and renal injury degree 
Table 5. The patient number enrolled and reached the endpoints.

\begin{tabular}{clccccc}
\hline Group & Enrolled and reached endpoints & $\mathbf{2 0 0 4}$ & $\mathbf{2 0 0 5}$ & $\mathbf{2 0 0 6}$ & $\mathbf{2 0 0 7}$ & $\mathbf{2 0 0 8}$ \\
\hline \multirow{4}{*}{ I } & Original patients & 0 & 60 & 80 & 77 & 70 \\
& Patients newly enrolled & 60 & 22 & 0 & 0 & 0 \\
& lost to follow-up & 0 & 0 & 0 & 2 & 3 \\
& Patients reached endpoints & 0 & 2 & 3 & 5 & 3 \\
& & & & & & \\
& Original patients & 0 & $59^{*}$ & $79^{*}$ & $75^{*}$ & $66^{*}$ \\
II & Patients newly enrolled & $59^{*}$ & $23^{*}$ & 0 & 0 & 0 \\
& lost to follow-up & 0 & 0 & 0 & $2^{*}$ & $2^{*}$ \\
& Patients reached endpoints & 0 & $3^{*}$ & $4^{*}$ & $7^{*}$ & $4^{*}$ \\
& & & & & & \\
& Original patients & 0 & 58 & 73 & $62^{\#}$ & $45^{\#}$ \\
III $\quad$ Patients newly enrolled & 58 & 24 & 0 & 0 & 0 \\
& lost to follow-up & 0 & 2 & 2 & 1 & 1 \\
& Patients reached endpoints & 0 & $7^{\#}$ & $9^{\#}$ & $16^{\#}$ & $14^{\#}$ \\
\hline
\end{tabular}

In group I, 13 patients reached endpoints (11 started dialysis, 2 used ACEI and ARB). In group II, 18 patients reached endpoints (15 started dialysis, 3 used ACEI and ARB; * $P>0.05$ group I versus group II). In group III, 46 patients reached endpoints (38 started dialysis, 8 used ACEI and ARB; ${ }^{\#} \mathrm{P}<0.05$ group I versus group III). Group I: Yan-Shen capsules group; group II: Niao-Du-Qing granules group; group III: placebo group.

Table 6. The ratio of patients needed erythropoietin (\%) (positive number/observed number).

\begin{tabular}{ccccc}
\hline Group & When enrolled & At end of 1st year & At end of 2nd year & At end of 3rd year \\
\hline I & $6(5 / 82)$ & $8.8(7 / 80)$ & $13(10 / 77)$ & $20(13 / 64)$ \\
II & $4.9(4 / 82)$ & $8.9(7 / 79)$ & $22(16 / 73)^{\star}$ & $41.7(25 / 60)^{\star \star \#}$ \\
III & $4.9(4 / 82)$ & $13.2(9 / 68)$ & $31.2(15 / 48)^{\star \star \# \#}$ & $70(21 / 30)^{\star \star \# \# \#}$ \\
\hline
\end{tabular}

Compared with group I, ${ }^{*} P<0.05,{ }^{* *} P<0.01,{ }^{* * *} P<0.001$. Compared with last year, ${ }^{\#} P<0.05,{ }^{\# \#} P<0.01$, $\# \#$ $\mathrm{P}<0.001$. Group I: Yan-Shen capsules group; group II: Niao-Du-Qing granules group; group III: placebo group.

Table 7. The variation of Scr $(\mu \mathrm{mol} / \mathrm{L})$ in three groups.

\begin{tabular}{ccccc}
\hline Group & Enrolled & At end of 1st year & At end of 2nd year & At end of 3rd year \\
\hline I & $205 \pm 42.1(n=82)$ & $229 \pm 52.0(n=80)$ & $241 \pm 62.1(n=77)$ & $282 \pm 68.3^{\#}(n=64)$ \\
II & $193 \pm 43.5(n=82)$ & $230 \pm 55.2(n=79)$ & $276 \pm 59.4(n=73)$ & $318 \pm 73.9^{\#}(n=60)$ \\
III & $189 \pm 40.3(n=82)$ & $304 \pm 63.5^{* \# \#}(n=68)$ & $423 \pm 69.1^{* * \# \# \#}(n=48)$ & $544 \pm 91.3^{* * * \# \# \#(n=30)}$ \\
\hline
\end{tabular}

Compared with group I, ${ }^{*} \mathrm{P}<0.05$, ${ }^{* *} \mathrm{P}<0.01,{ }^{* * *} \mathrm{P}<0.001$. Compared with the time when enrolled, ${ }^{*} \mathrm{P}<$ $0.05,{ }^{\# \#} \mathrm{P}<0.01,{ }^{\# \#} \mathrm{P}<0.001$. Group I: Yan-Shen Capsules Group; group II: Niao-Du-Qing Granules group; group III: placebo group.

of the three groups were comparable.

The number of the Yan-Shen capsule group's patients who reached the endpoints was significantly smaller than that of group III $(p<0.05)$ and group II, although no statistically significant difference for the latter was found $(p>0.05)$. Our results suggest that both Yan-Shen capsules and Niao-Du-Qing granules had significant protective effects on the residual renal function of advanced CKD patients due to CGN, LN, and Autosomal dominant polycystic kidney disease (ADPKD). Yan-Shen capsules may be superior to Niao-Du-Qing granules in terms of protecting advanced CKD patients from progressive renal damage. No obvious side effects of Yan-Shen capsules and Niao-Du-Qing granules were found during our observation. These data suggest that Yan-Shen capsules and Niao-Du-Qing granules are safe to use in treating advanced CKD patients and have good compliance.

The annual loss of GFR in CKD patients was highly disparate (from 1 to $13 \mathrm{ml} / \mathrm{min} / 1.73 \mathrm{~m}^{2}$ ) among different reports (Jones et al., 2006; Ruggenenti et al., 2008), depending on the patient's age, primary disease, 
Table 8. The variation of BUN (mmol/L) in three groups.

\begin{tabular}{ccccc}
\hline Group & When enrolled & At end of 1st year & At end of 2nd year & At end of 3rd year \\
\hline I & $12.5 \pm 4.3(n=82)$ & $15.4 \pm 5.9(n=80)$ & $18.2 \pm 6.1(n=77)$ & $19.2 \pm 9.3^{\# \#}(n=64)$ \\
II & $12.2 \pm 4.4(n=82)$ & $15.6 \pm 5.7(n=79)$ & $17.9 \pm 6.4 \quad(n=73)$ & $18.3 \pm 9.9^{\#}(n=60)$ \\
III & $11.9 \pm 4.1(n=82)$ & $15.9 \pm 5.4(n=68)$ & $21.3 \pm 6.7^{* \#}(n=48)$ & $26.1 \pm 9.3^{\star \# \# ~}(n=30)$ \\
\hline
\end{tabular}

Note: Compared with group I, ${ }^{*} \mathrm{P}<0.05$. Compared with the time when enrolled, ${ }^{\#} \mathrm{P}<0.05,{ }^{\# \#} \mathrm{P}<0.01$. Group I: Yan-Shen Capsules Group; group II: Niao-Du-Qing Granules group; group III: placebo group.

Table 9. The variation of GFR $\left(\mathrm{ml} / \mathrm{min} / 1.73 \mathrm{~m}^{2}\right)$ in three groups.

\begin{tabular}{ccccc}
\hline Group & Enrolled & At end of 1st year & At end of 2nd year & At end of 3rd year \\
\hline I & $33.4 \pm 4.2(n=82)$ & $29.3 \pm 5.2(n=80)$ & $27.7 \pm 4.2^{\#}(n=77)$ & $23.1 \pm 4.3^{\# \#}(n=64)$ \\
II & $35.5 \pm 4.9(n=82)$ & $29.2 \pm 5.5(n=79)$ & $25.1 \pm 5.1^{\#}(n=73)$ & $20.1 \pm 4.9^{\# *}(n=60)$ \\
III & $35.6 \pm 5.3(n=82)$ & $27.1 \pm 5.5^{\#}(n=68)$ & $20.1 \pm 5.1^{\# \# * \star}(n=48)$ & $14.4 \pm 3.7^{\# \# \#_{\star \star \star *}(n=30)}$ \\
\hline
\end{tabular}

Note: Compared with group I, ${ }^{*} P<0.05,{ }^{* *} P<0.01,{ }^{* * *} P<0.001$. Compared with the time when enrolled, ${ }^{\#} P<0.05$, ${ }^{\# \#} P<$

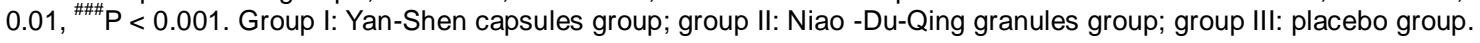

Table 10. The variation of blood pressure $(\mathrm{mmHg})$ in three groups (Hypertension number/observed number).

\begin{tabular}{ccccc}
\hline Group & When enrolled & At end of 1st year & At end of 2nd year & At end of 3rd year \\
\hline I & $164.5 / 104.1(n=48 / 82)$ & $145.4 / 95.4(n=50 / 80)$ & $146.1 \pm / 90.1(n=50 / 77)$ & $146.7 / 90.9(n=51 / 66)$ \\
II & $165.4 / 103.9(n=45 / 82)$ & $141.4 / 94.8(n=46 / 79)$ & $147.4 / 91.3(n=47 / 73)$ & $140.3 / 89.6(n=48 / 60)$ \\
III & $165.9 / 104.2(n=46 / 82)$ & $150.6 / 95.7(n=44 / 75)$ & $148.8 / 86.2(n=41 / 68)$ & $149.6 / 97.1(n=37 / / 46)$ \\
\hline
\end{tabular}

Note: P > 0.05 (group I versus group II, group I versus group III). Group I: Yan-Shen capsules group; group II: Niao -Du-Qing granules group; group III: placebo group.

complications, urinary protein, therapy, and stage of disease. GFR loss became more rapid as the disease progressed. For example, in an advanced CKD patient with a SCr level of about $400 \mu \mathrm{mol} / \mathrm{L}$, a common cold can increase the SCr to $707 \mu \mathrm{mol} / \mathrm{L}$. Despite the fact that all patients had advanced (stage III to IV) CKD, the annual GFR decrease in the Yan-Shen capsules group and NiaoDu-Qing granules group (3.4 and $5.1 \mathrm{ml} / \mathrm{min} / 1.73 \mathrm{~m}^{2}$, respectively) was limited to an acceptable rate and was significantly smaller than that in the placebo group (7.2 $\mathrm{ml} / \mathrm{min} / 1.73 \mathrm{~m}^{2}$ ), which showed the efficacy of the two traditional Chinese medicines in delaying advanced CKD progression to ESRD.

The Yan-Shen capsule group had significantly fewer patients who needed erythropoietin than the placebo and Niao-Du-Qing granules groups. This result suggests that Yan-Shen capsule is also capable of modifying anaemia in addition to its protective role in residual renal function. ACEI, ARB, keto acids and steroids demonstrated protective effects on the residual renal function of CKD patients.

Therefore, to avoid interference, the patients enrolled in our study did not use these drugs. In clinical practice, these drugs could be taken by CKD patients along with Yan-Shen capsules or Niao-Du-Qing granules for better efficacy, according to the individual's condition. Some patients of the three groups manifested uncontrolled hypertension and had to use ACEI and/or ARB; these patients were also included in the group which reached the secondary endpoint, although they did not enter ESRD.

\section{Conclusion}

This study demonstrated that both Yan-Shen capsules and Niao-Du-Qing granules had significant protective effects on residual renal function in advanced CKD, thereby delaying the progression to dialysis without obvious side effects, compared with placebo. Yan-Shen capsules may be superior to Niao-Du-Qing granules in protecting residual renal function and modifying anaemia.

\section{REFERENCES}

Cai Q, Li XM, Wang HY (2001). Astragali and angilica protect the kidney against ischemia and reperfusion injury and accelerate recovery. Chin. Med. J. 114:119-123.

Chen JL, Chen YC, Yang SH, Ko YF, Chen SY (2009). Immunological alterations in lupus-prone autoimmune (NZB/NZW) F1 mice by mycelia Chinese medicinal fungus Cordyceps sinensis-induced redistributions of peripheral mononuclear $T$ lymphocytes. Clin. Exp. Med. 9:277-284. 
Enyu I (2006). Is ACEI Superior to ARB for CKD? Int. Med. 45:179-181. Guo XH, Liu ZH, Dai CS (2001). The effect of rhein on the hypertrophy of renal tubular epithelial cell and accumulation of extracellular matrix induced by transforming growth factor $\beta$. Chin. J. Nephrol. Dial. Transplant. 10:101-105.

Hamlett L, Haragsim L (2007). Quotidian hemodialysis and inflammation associated with chronic kidney disease. Adv. Chronic Kidney Dis. 14:e35-e42.

Hou FF, Zhang X, Zhang GH, Xie D, Chen PY, Zhang WR, Jiang JP, Liang M, Wang GB, Liu ZR, Geng RW (2006). Efficacy and safety of benazepril for advanced chronic renal insufficiency. N. Engl. J. Med. 354:131-140.

Jinous A, Fereshteh R (2012). Chemistry, pharmacology and medicinal properties of Peganum harmala L. Afr. J. Pharm. Pharmacol. 6:15731580.

Jones C, Roderick P, Harris S, Rogerson M (2006). Decline in kidney function before and after nephrology referral and the effect on survival in moderate to advanced chronic kidney disease. Nephrol. Dial. Transplant 21:2133-2143.

Levey AS, Coresh J, Greene T, Marsh J, Stevens LA, Kusek JW, Van Lente $F$ (2007). Expressing the Modification of Diet in Renal Disease Study equation for estimating glomerular filtration rate with standardized serum creatinine values. Clin. Chem. 53:766-772.

Muhammad A, Ghiasuddin, Anwar S, Naveed M, Ashfaq AK, Bina SS (2012). Evaluation of Viburnum grandiflorum for its in-vitro pharmacological screening. Afr. J. Pharm. Pharmacol. 6:1606-1610.

National Kidney Foundation (2002). K/DOQI clinical practice guidelines for chronic kidney disease: Evaluation, classification, and stratification. Am. J. Kidney Dis. 39:S1-S266.

Philipneri MD, Rocca RLA, Schnitzler MA, Abbott KC, Brennan DC, Takemoto SK, Buchanan PM, Burroughs TE, Willoughby LM, Lentine $\mathrm{KL}$ (2008). Delivery patterns of recommended chronic kidney disease care in clinical practice: Administrative claims-based analysis and systematic literature review. Clin. Exp. Nephrol. 12:41-52.

Ruggenenti P, Perticucci E, Cravedi P, Gambara V, Costantini M, Sharma SK, Perna A, Remuzzi G (2008). Role of remission clinics in the longitudinal treatment of CKD. J. Am. Soc. Nephrol. 19:12131224.

Sima N, Mahdieh A, Narges K (2012). Evaluation of analgesic andantiinflammatory effects of fresh onion juice in experimental animals. Afr. J. Pharm. Pharmacol. 6:1679-1684.
Thorp ML, Eastman L, Smith DH, Johnson ES (2006). Managing the burden of chronic kidney disease. Dis. Manag. 9:115-121.

Wu HL, Lin HC, Ruan XL (2004). Observation of the Curative Efficiency of Uremic Clearance Granules in 118 Patients with Chronic Renal Failure. Chin. J. Integr. TCM-WM Nephrol. 5:21-24.

Yuan FH, Guang LX, Li YB (2004). Effect of Yan-Shen capsule on mesangial cell apoptosis and extracellular matrix metabolism of remnant kidney. China J. Mod. Med. 14:5-10.

Yuan FH, Guang LX, Liao LS (1998). The experimental study for mechanism of retarding the lesion progress of remnant kidney by Yan-Shen capsule. Am. J. Orient. Med. Res. 1:151-152.

Zhang LX, Zuo L, Xu GB (2006). Community based screening for chronic kidney disease among population older than 40 years in Beijing. Chin. J. Nephrol. 22:67-71.

Zheng JM, Zhu JM, Li LS, Liu ZH (2008). Rhein reverses the diabetic phenotype of mesangial cells over-expressing the glucose transporter (GLUT1) by inhibiting the hexosamine pathway. Br. J. Pharmacol. 153:1456-1464.

Zhu W, Wang XM (2005). Progress in Study on Mechanisms of Rhubarb in Treating Chronic Renal Failure. Chin. J. Integr. West Med. 25:471475 . 